\title{
EQUATIONAL BASES FOR SUBVARIETIES OF DOUBLE MS-ALGEBRAS
}

\author{
by T. S. BLYTH ${ }^{1}$, A. S. A. NOOR ${ }^{2}$, AND J. C. VARLET ${ }^{1}$
}

(Received 31 March 1987)

1. Introduction. An $M S$-algebra is an algebra $\left(L ; \vee, \wedge,{ }^{\circ}, 0,1\right)$ of type $(2,2,1,0,0)$ such that $(L ; \vee, \wedge, 0,1)$ is a distributive lattice with smallest element 0 and greatest element 1 , and $x \mapsto x^{\circ}$ is a unary operation such that $1^{\circ}=0, x \leq x^{\circ}$ for all $x \in L$, and $(x \wedge y)^{\circ}=x^{\circ} \vee y^{\circ}$ for all $x, y \in L$. These algebras belong to the class of Ockham algebras introduced by Berman [3]; see also $[2,10,15]$. A double $M S$-algebra is an algebra $\left(L, \vee, \wedge,{ }^{\circ},+, 0,1\right)$ of type $(2,2,1,1,0,0)$ such that $\left(L,^{\circ}\right)$ and $\left(L_{d},{ }^{+}\right)$are MS-algebras, where $L_{d}$ denotes the dual of $L$, and the operations ${ }^{\circ}$, ${ }^{+}$are linked by the identities $x^{\circ+}=x^{\circ}$ and $x^{+\circ}=x^{++}$. We refer to $[5,6,7,8]$ for the basic properties of MS-algebras and double MS-algebras. Concerning the latter, the properties $x^{000}=x^{\circ}, x^{+++}=x^{+}$, and $x^{\circ} \leq x^{+}$will be used frequently. The class of double MS-algebras is congruencedistributive and consequently the results of [13] can be applied. As to general results in lattice theory and universal algebra, the reader may consult $[1,9,12]$.

In [8] we gave a complete description of the 22 non-isomorphic subdirectly irreducible double MS-algebras. We can order these algebras by writing $A \leq B$ if and only if $A$ is isomorphic to a subalgebra of $B$. In so doing, we obtain the Hasse diagram of Figure 1 in which $\mathbf{n}$ denotes the subdirectly irreducible algebra $S I_{n}$.

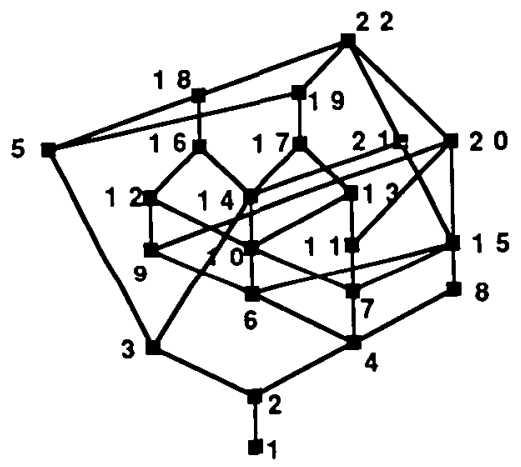

Figure 1.

The lattice of subvarieties of double MS-algebras can in theory be obtained from this by applying a theorem of Davey [11] in precisely the same way as we did in [6] to obtain the lattice of subvarieties of MS-algebras. However, in the case of double MS-algebras

\footnotetext{
${ }^{1}$ NATO Research Grant $85 / 0532$ is gratefully acknowledged.

${ }^{2}$ A Commonwealth Staff Research Fellowship at the University of St Andrews is gratefully acknowledged.
}

Glasgow Math. J. 31 (1989) 1-16. 
the lattice is rather large and so we shall concentrate on some important ideal sublattices. Specifically, if $\mathbf{S I}_{n}$ denotes the subvariety generated by $S I_{n}$, we shall describe here the lattices of subvarieties of $\mathbf{S I}_{21}, \mathbf{S I}_{20}$ and $\mathbf{S I}_{18} \vee \mathbf{S I}_{19}$. We shall also obtain equational bases for the subvarieties in these lattices, and hence in particular for all of the subvarieties generated by the 22 subdirectly irreducible double MS-algebras. It is a pleasure for the third author to acknowledge stimulating conversations on this topic with Pierre Goossens.

2. Semisimple double MS-algebras. Of the 22 non-isomorphic subdirectly irreducible double MS-algebras listed in [8], 11 are simple. These comprise the subset

$$
\left\{S I_{n} ; n=1,2,3,4,6,7,8,10,14,15,21\right\},
$$

which forms an ideal of the ordered set of Fig. 1. A double MS-algebra is said to be semisimple if it is a subdirect product of simple double MS-algebras; in other words, if it is a subdirect product of copies of $S I_{21}$.

We can characterise the semisimple double MS-algebras as follows, in which $\Phi_{+}^{\circ}$ is the congruence

$$
(x, y) \in \Phi_{+}^{\circ} \Leftrightarrow\left(x^{\circ}=y^{\circ} \text { and } x^{+}=y^{+}\right) .
$$

THEOREM 2.1. The following conditions on a double MS-algebra are equivalent:

$\left(\alpha_{0}\right) L$ is semisimple;

$(\alpha)(\forall x, y \in L) \quad x \wedge y^{\infty} \leq x^{++} \vee y$;

$\left(\alpha_{1}\right) \Phi_{+}^{\circ}=\omega$.

Proof. $\left(\alpha_{0}\right) \Rightarrow(\alpha)$ : It suffices to observe that $S I_{21}$ satisfies $(\alpha)$.

$(\alpha) \Rightarrow\left(\alpha_{0}\right)$ : Examination of each of the subdirectly irreducible double MS-algebras reveals that only those that are simple satisfy $(\alpha)$. Thus, if $L$ satisfies $(\alpha)$ then by Birkhoff's Theorem $L$ is a subdirect product of copies of $S I_{21}$.

$(\alpha) \Rightarrow\left(\alpha_{1}\right)$ : Suppose that $L$ satisfies $(\alpha)$ and that $x, y \in L$ are such that $x^{\circ}=y^{\circ}$ and $x^{+}=y^{+}$. Then $(\alpha)$ gives $x=x \wedge x^{\infty}=x \wedge y^{\infty} \leq x^{++} \vee y=y^{++} \vee y=y$. Similarly we obtain $y \leq x$, whence $y=x$.

$\left(\alpha_{1}\right) \Rightarrow(\alpha)$ : Suppose that $\Phi_{+}^{\circ}=\omega$ and consider the elements

We have

$$
p=x \wedge y^{\infty}, \quad q=x \wedge y^{\infty} \wedge\left(x^{++} \vee y\right) \text {. }
$$

$$
\begin{aligned}
& q^{\circ}=x^{\circ} \vee y^{\circ} \vee\left(x^{+} \wedge y^{\circ}\right)=x^{\circ} \vee y^{\circ}=p^{\circ} \\
& q^{+}=x^{+} \vee y^{\circ} \vee\left(x^{+} \wedge y^{+}\right)=x^{+} \vee y^{\circ}=p^{+} .
\end{aligned}
$$

It follows that $q=p$ whence $(\alpha)$ follows.

We shall now obtain the lattice of subvarieties of semisimple double MS-algebras and equational bases for the subvarieties in this lattice. We can construct the lattice in question by applying Davey's Theorem [11] in precisely the same way as we did in [6]. We can predict the size of the this lattice by means of the following ingenious result of Berman and Köhler [4]. 
THEOREM. Let $F$ be a finite ordered set and let $O(F)$ be its lattice of order ideals. Then, for every $x \in F$,

$$
|O(F)|=|O(F \backslash\{x\})|+\left|O\left(F \backslash C_{x}\right)\right|
$$

where $C_{x}=\{y \in L ; y \leq x$ or $y \geq x\}$.

Applying this to the ideal generated by $S I_{21}$ in the Hasse diagram of Fig. 1, we see that the lattice of subvarieties of semisimple double MS-algebras has 31 elements. By Davey's Theorem, the lattice itself is that depicted in Fig. 2 (ignore for the moment the other labelling).

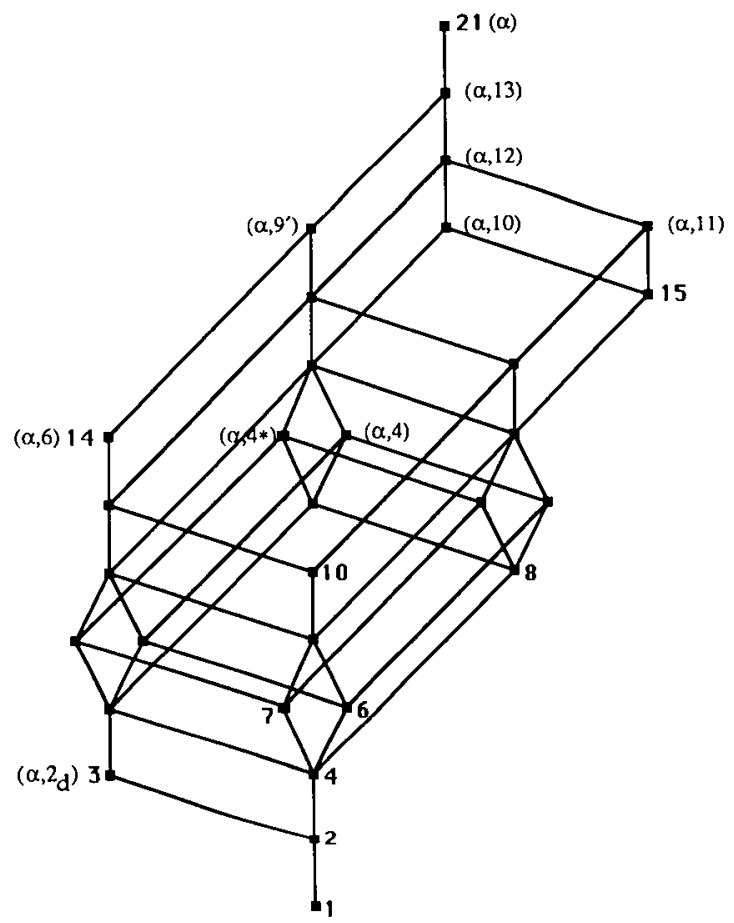

Figure 2.

As to the problem of establishing equational bases for each of the subvarieties in this lattice, our strategy will be to discover first equational bases for its $\wedge$-irreducible elements. Equational bases for all the subvarieties can then be deduced from these.

For this purpose, a few preliminary considerations are necessary. Note first that

$$
S I_{1}, S I_{2}, S I_{3}, S I_{4}, S I_{5}, S I_{8}, S I_{10}, S I_{14}, S I_{15}, S I_{20}, S I_{21}, S I_{22}
$$

are self-dual, whereas we have the dual isomorphisms

$$
S I_{6} \stackrel{\mathrm{d}}{=} S I_{7}, S I_{9} \stackrel{\mathrm{d}}{\simeq} S I_{11}, S I_{12} \stackrel{\mathrm{d}}{\simeq} S I_{13}, S I_{16} \stackrel{\mathrm{d}}{\simeq} S I_{17}, S I_{18} \stackrel{\mathrm{d}}{\simeq} S I_{19} \text {. }
$$


For a given axiom $(n)$ we shall denote by $\left(n^{\star}\right)$ the axiom obtained from $(n)$ by replacing $\vee, \wedge,{ }^{\circ},+, 0,1, \leq$ respectively by $\wedge, \vee,{ }^{+},{ }^{\circ}, 1,0, \geq$. If $(n) \Leftrightarrow\left(n^{\star}\right)$ then we shall say that $(n)$ is self-dual. Clearly, if $L_{i}$ satisfies $(n)$ and $L_{j} \stackrel{\mathrm{d}}{=} L_{i}$ then $L_{j}$ satisfies $\left(n^{\star}\right)$.

We shall require the following facts concerning axioms of the form $x^{p} \wedge x^{q} \leq y^{r} \vee y^{s}$ where $\{p, q, r, s\} \subseteq\left\{{ }^{1},{ }^{\circ},{ }^{\infty},{ }^{+},{ }^{++}\right\}$and $x^{1}$ means $x$. Such axioms will be considered trivial if either side reduces to a single term, e.g. $x \wedge x^{\infty}=x$.

THEOREM 2.2. Inequalities of the form
(i) $f \leq y^{++} \vee y^{+}$,
(ii) $f \leq y \vee y^{+}$,
(iii) $f \leq y^{\circ \circ} \vee y^{+}$,
(iv) $f \leq y^{\circ} \vee y^{\circ}$

are equivalent. Likewise, inequalities of the form
(j) $x^{\circ} \wedge x^{\circ} \leq g$
(jj) $x \wedge x^{\circ} \leq g$
(jjj) $x^{++} \wedge x^{\circ} \leq g$,
(jw) $x^{++} \wedge x^{+} \leq g$

are equivalent.

Proof. It is clear that $(i) \Rightarrow($ ii $) \Rightarrow$ (iii). That (iii) $\Rightarrow(i)$ follows on writing $y^{++}$for $y$. To see that $(i) \Rightarrow(i v)$, write $y^{\circ \circ}$ for $y$; and that $(i v) \Rightarrow(i)$ write $y^{++}$for $y$. The second statement is similar.

In what follows we shall denote by $S(L)$ the skeleton of $L$, i.e. the set

$$
\left\{x \in L ; x=x^{\circ}\right\}=\left\{x \in L ; x=x^{++}\right\}=\left\{x \in L ; x^{\infty}=x^{++}\right\} .
$$

THEOREM 2.3. Inequalities of the form

$$
\text { (v) } y \vee y^{\circ} \geq f \in S(L), \quad \text { (vi) } y^{++} \vee y^{\circ} \geq f \in S(L)
$$

are equivalent. Likewise, inequalities of the form

$$
(w) x \wedge x^{+} \leq g \in S(L), \quad(w j) x^{\infty} \wedge x^{+} \leq g \in S(L)
$$

are equivalent.

Proof. It suffices to apply ${ }^{++}$and ${ }^{\infty 0}$ respectively.

THEOREM 2.4. There are 36 non-trivial axioms of the form $x^{p} \wedge x^{q} \leq y^{r} \vee y^{s}$. Of these, 16 are equivalent to

16 are equivalent to

$$
\text { (a) } x \wedge x^{\circ} \leq y^{\circ \circ} \vee y^{\circ} \text {, }
$$

3 are equivalent to

$$
\text { (b) } x \wedge x^{\circ} \leq y \vee y^{\circ} \text {, }
$$

and the remaining axiom is

$$
\text { (c) } x^{\circ 0} \wedge x^{+} \leq y^{++} \vee y^{\circ} \text {, }
$$

$$
\text { (d) } x \wedge x^{+} \leq y \vee y^{\circ} \text {. }
$$

Proof. That there are 36 non-trivial forms is clear from the fact each of $x^{\circ}, x^{+}$can be taken along with any one of $x^{++}, x, x^{\infty}$. Now by Theorem 2.2 both sides of $(a)$ may take 4 different forms. As for (b); this is axiom (5) of [6] which is self dual and hence 
equivalent to

$$
\left(b^{\star}\right) x \wedge x^{+} \leq y \vee y^{+} \text {. }
$$

By Theorem 2.2, (b) has 4 equivalent forms for the left-hand side, three of which belong to $S(L)$. Then, by Theorem 2.3, there are 2 equivalent forms for the right-hand side. Similarly, there are 8 equivalent forms of $\left(b^{\star}\right)$. As for $(c)$, Theorem 2.3 provides 3 equivalent forms. It follows from the above count that the only remaining axiom is $(d)$.

Equational bases for each of the subvarieties in the class of MS-algebras may be found in [6]. They also appear in a more general setting in [15]. As all of the axioms that are involved there will play an important rôle in what follows, we list them here for convenience.

$$
\begin{array}{ll}
\text { (1) } x=y & \\
\text { (2) } x \vee x^{\circ}=1 & \text { (5) } x \wedge x^{\circ} \leq y \vee y^{\circ} \\
\text { (2 }) x \wedge x^{\circ}=0 & \text { (6) } x \wedge x^{\circ} \leq y^{\circ} \vee y^{\circ} \\
\text { (3) } x=x^{\circ} & \text { (7) }\left(x \wedge x^{\circ}\right) \vee y \vee y^{\circ}=\left(x^{\circ} \wedge x^{\circ}\right) \vee y \vee y^{\circ} \\
\text { (4) } x \wedge x^{\circ}=x^{\circ} \wedge x^{\circ} & \text { (8) } x \vee y^{\circ} \vee y^{\circ}=x^{\circ} \vee y^{\circ} \vee y^{\circ} \\
\text { (4d } x \vee x^{\circ}=x^{\infty} \vee x^{\circ} & \text { (9) }\left(x \wedge x^{\circ}\right) \vee y^{\circ} \vee y^{\circ \circ}=\left(x^{\circ} \wedge x^{\circ}\right) \vee y^{\circ} \vee y^{\circ}
\end{array}
$$

The complete list of implications between these identities was given in [6, Theorem 2.2]. In [7] we showed that if $L$ is a double MS-algebra then (8) $\Rightarrow(7)$, and the axioms (1), $(2),\left(2_{d}\right),(3),(5),(6),(7)$ are self-dual. Recently, Noor [14] succeeded in showing that (9) is also self-dual.

Observe from [6] that (4) is equivalent to $\left(8_{d}\right)$ and so the axiom

$$
\left(4^{\star}\right) x \vee x^{+}=x^{++} \vee x^{+}
$$

is equivalent to $\left(8_{d}^{\star}\right)$ which is equivalent to $(8)$.

Also, (9) is equivalent to

$$
\left(9^{\prime}\right) x^{\circ} \wedge x^{\circ} \wedge y^{+} \leq y^{\circ} \vee y^{\circ \circ}
$$

Indeed, applying ${ }^{+}$to (9) gives

$$
\left(x^{+} \vee x^{\circ}\right) \wedge y^{\circ \circ} \wedge y^{\circ}=\left(x^{\circ} \vee x^{\circ}\right) \wedge y^{\circ} \wedge y^{\circ},
$$

whence $x^{+} \wedge y^{\circ} \wedge y^{\circ} \leq x^{\circ} \vee x^{\circ}$ and (9') follows on interchanging $x$ and $y$. Conversely, applying ${ }^{\circ}$ to $\left(9^{\prime}\right)$ yields

$$
x^{\circ \circ} \vee x^{\circ} \vee y^{++} \geq y^{\circ \circ} \wedge y^{\circ}
$$

Since also $x^{\circ \circ} \vee x^{\circ} \vee y^{\circ} \geq y^{\circ \circ} \wedge y^{\circ}$ it follows that $x^{\circ \circ} \vee x^{\circ} \vee\left(y^{++} \wedge y^{\circ}\right) \geq y^{\circ \circ} \wedge y^{\circ}$ and so

$$
x^{\circ} \vee x^{\circ} \vee\left(y^{++} \wedge y^{\circ}\right) \geq x^{\circ \circ} \vee x^{\circ} \vee\left(y^{\circ \circ} \wedge y^{\circ}\right) \text {. }
$$

Consequently $x^{\circ \circ} \vee x^{\circ} \vee\left(y \wedge y^{\circ}\right) \in S(L)$, from which (9) follows on interchanging $x$ and $y$. 
In this section we shall consider the following inequalities. They have, of course, been chosen with a considerable amount of hindsight.

$$
\begin{aligned}
& \text { (1), }\left(2_{d}\right),(4),\left(4^{\star}\right),(6),\left(9^{\prime}\right), \\
& \text { (10) } x^{\circ} \wedge x^{\infty} \wedge y^{+} \leq x^{++} \vee y^{\circ}, \\
& \text { (11) } x^{+} \wedge x^{\infty} \wedge y^{+} \leq x^{++} \vee y^{\circ} \vee y^{++}, \\
& \text {(12) } x^{\circ} \wedge x^{\infty} \wedge y^{+} \leq x^{++} \vee y^{\circ} \vee y^{++}, \\
& \text {(13) } x^{\circ} \wedge x^{\infty} \wedge y^{+} \leq x^{++} \vee y^{\circ} \vee y^{\infty}, \\
& \text { (a) } x \wedge y^{\infty} \leq x^{++} \vee y .
\end{aligned}
$$

Note that relation (10) is self-dual. To see this, apply ${ }^{\circ}$ to each side of (10) then interchange $x$ and $y$ to obtain $x^{\infty \circ} \wedge y^{+} \leq y^{\circ} \vee y^{\circ} \vee x^{++}$. Now write $y \wedge x^{+}$for $y$ to obtain

$$
x^{\infty} \wedge\left(y^{+} \vee x^{++}\right) \leq\left(y^{\infty} \wedge x^{+}\right) \vee\left(y^{\circ} \vee x^{++}\right) \vee x^{++},
$$

from which $\left(10^{\star}\right)$ follows. The reverse implication is established similarly.

Also, (11) is self-dual. This follows from the fact that (11) is equivalent to $x^{\infty} \wedge x^{+} \leq x^{++} \vee x^{\circ}$. Indeed, taking $y=x$ in (11) we obtain this relation; and (11) follows from this relation on writing $x \vee y$ for $x$.

By Theorem 2.1, the relation $(\alpha)$ describes the subvariety $\mathbf{S I}_{21}$. Observe now that, the ordering being logical implication, we have the Hasse diagram of Figure 3.

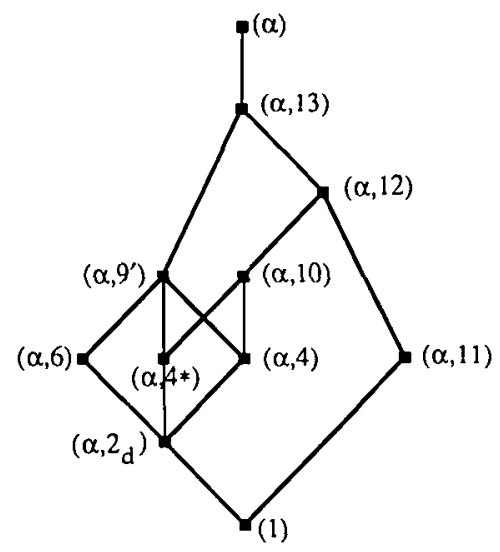

Figure 3.

Of the implications in Figure 3, only the following is non-trivial:

(4) $\Rightarrow(10)$ : By (4) we have $x^{\infty} \wedge x^{\circ} \leq x$ so $x^{\infty} \wedge x^{\circ} \leq x^{++}$and hence $x^{\circ} \wedge x^{\infty} \wedge y^{+} \leq$ $x^{++} \vee y^{\circ}$. 
THEOREM 2.5. In the lattice of subvarieties of semisimple double MS-algebras the $\wedge$-irreducible elements have the following equational bases:

$$
\begin{array}{ll}
\mathbf{S I}_{1}:(1), & \mathbf{S I}_{3}:\left(\alpha, 2_{d}\right) \\
\mathbf{S I}_{3} \vee \mathbf{S I}_{6} \vee \mathbf{S I}_{8}:(\alpha, 4), & \mathbf{S I}_{3} \vee \mathbf{S I}_{7} \vee \mathbf{S I}_{8}:\left(\alpha, 4^{\star}\right), \\
\mathbf{S I}_{14}:(\alpha, 6), & \mathbf{S I}_{8} \vee \mathbf{S I}_{14}:\left(\alpha, 9^{\prime}\right), \\
\mathbf{S I}_{3} \vee \mathbf{S I}_{15}:(\alpha, 10), & \mathbf{S I}_{10} \vee \mathbf{S I}_{15}:(\alpha, 11), \\
\mathbf{S I}_{3} \vee \mathbf{S I}_{10} \vee \mathbf{S I}_{15}:(\alpha, 12), & \mathbf{S I}_{14} \vee \mathbf{S I}_{15}:(\alpha, 13), \\
\mathbf{S I}_{21}:(\alpha) . &
\end{array}
$$

Proof. We refer to Fig. 2 in which the $\wedge$-irreducible elements have been labelled accordingly. Observe that in this lattice the ordered set of $\wedge$-irreducible elements is isomorphic to the ordered set of Fig. 3. Also, it is readily verified that the strongest of the above relations that are satisfied by each of the $\wedge$-irreducible elements are as listed in the statement of the theorem. It follows that the lattice of relations whose set of $\wedge$-irreducible elements is that of Fig. 3 is isomorphic to the lattice of Fig. 2 and the quational bases are as asserted.

It should be remarked that Theorem 2.5 can also be established by considering each $\wedge$-irreducible element in turn. For example, consider the relation (10). This is not satisfied by $S I_{21}$. But, as can readily be verified, it is satisfied by $S I_{14}$ and $S I_{15}$. It follows that an equational basis for $\mathbf{S I}_{14} \vee \mathbf{S I}_{15}$ is $(\alpha, 10)$. Indeed, it was this kind of consideration that led to the above list of relations. The advantage of having equational bases for the $\wedge$-irreducible elements lies in the fact that we can use them to obtain equational bases for all of the subvarieties in the lattice. This we do first for the subvarieties generated by the subdirectly irreducibles, and for this purpose we list the following additional axioms:

$$
\begin{aligned}
& \text { (14): } x^{\infty} \wedge y^{+} \leq x^{++} \vee y^{\circ}, \\
& \text { (15): } x^{\infty} \wedge x^{+} \leq y^{++} \vee y^{\circ}, \\
& \text { (16): } x \wedge x^{+} \leq y \vee y^{\circ} .
\end{aligned}
$$

COROLLARY 2.6. An equational basis for $\mathbf{S I}_{15}$ is $(\alpha, 14)$.

Proof. It is clear that (14) implies both (10) and (11), so it suffices to show that (10) and (11) together imply (14). For this purpose, observe that writing $x \wedge y^{++}$for $x$ in (10) gives

and therefore

$$
\left(x^{\circ} \vee y^{+}\right) \wedge x^{\infty} \wedge y^{++} \wedge y^{+} \leq\left(x^{++} \wedge y^{++}\right) \vee y^{\circ}
$$

$$
x^{\infty} \wedge y^{++} \wedge y^{+} \leq x^{++} \vee y^{\circ} .
$$

Also, by (11) we have $x^{+} \wedge x^{\infty} \leq x^{++} \vee x^{\circ}$ and so

$$
x^{+} \wedge\left(x^{\circ} \vee x^{\circ}\right)=x^{+} \wedge\left(x^{\circ} \vee x^{++}\right) \text {. }
$$

Now applying ${ }^{\circ}$ to (10) we obtain $x^{+} \wedge y^{\circ} \leq x^{\circ} \vee x^{\circ} \vee y^{++}$. Interchanging $x, y$ in this we 
have, making use of the above observations,

as required.

$$
\begin{aligned}
x^{\circ \circ} \wedge y^{+} & =x^{\circ \circ} \wedge y^{+} \wedge\left(x^{++} \vee y^{\circ} \vee y^{\circ \circ}\right) \\
& =\left(x^{++} \wedge y^{+}\right) \vee\left[x^{\circ \circ} \wedge y^{+} \wedge\left(y^{\circ} \vee y^{\circ 0}\right)\right] \\
& =\left(x^{++} \wedge y^{+}\right) \vee\left[x^{\circ \circ} \wedge y^{+} \wedge\left(y^{\circ} \vee y^{++}\right)\right] \\
& =\left(x^{++} \wedge y^{+}\right) \vee\left(x^{\circ 0} \wedge y^{\circ}\right) \vee\left(x^{\circ \circ} \wedge y^{+} \wedge y^{++}\right) \\
& \leq x^{++} \vee y^{\circ} \vee x^{++} \vee y^{\circ} \\
& =x^{++} \vee y^{\circ},
\end{aligned}
$$

Corollary 2.7. An equational basis for $\mathbf{S I}_{8}$ is (3).

Proof. It suffices by Corollary 2.6 to show that (4), (4^) and (14) together imply (3). Now, taking $y=x$ in (14) gives $x^{\circ \circ} \wedge x^{+} \leq x^{++} \vee x^{\circ}$ and so, by (4),

$$
\begin{aligned}
x^{\circ \circ} \wedge x^{+} & =x^{\circ} \wedge x^{+} \wedge\left(x^{++} \vee x^{\circ}\right) \\
& =\left(x^{++} \wedge x^{+}\right) \vee\left(x^{\circ \circ} \wedge x^{\circ}\right) \\
& =\left(x^{++} \wedge x^{+}\right) \vee\left(x \wedge x^{\circ}\right) .
\end{aligned}
$$

Applying ${ }^{++}$to this we obtain $x^{\circ 0} \wedge x^{+}=x^{++} \wedge x^{+}$and so $x \wedge x^{+} \in S(L)$. But by (4*) we have $x \vee x^{+} \in S(L)$. Since $S(L)$ is semiconvex [in the sense that if $a \vee b \in S(L)$ and $a \wedge b \in S(L)$ then $a, b \in S(L)$ ], it follows that $x \in S(L)$, whence we have (3).

COROLlaRY 2.8. An equational basis for $\mathbf{S I}_{10}$ is $(\alpha, 15)$.

Proof. It suffices to show that (6) and (11) together imply (15). Now from (11) we have $x^{\circ \circ} \wedge x^{+} \leq x^{++} \vee x^{\circ}$ and so

$$
x^{\circ 0} \wedge x^{+}=x^{\circ 0} \wedge x^{+} \wedge\left(x^{++} \vee x^{\circ}\right)=\left(x^{++} \wedge x^{+}\right) \vee\left(x^{\circ \circ} \wedge x^{\circ}\right)
$$

By Theorem 2.2, (6) is equivalent to $x^{++} \wedge x^{+} \leq y^{\circ \circ} \vee y^{\circ}$ and also to $x^{\circ \circ} \wedge x^{\circ} \leq y^{\circ \circ} \vee y^{\circ}$. Thus we see that $x^{\circ \circ} \wedge x^{+} \leq y^{\circ \circ} \vee y^{\circ}$, which is equivalent to $x^{\circ 0} \wedge x^{+} \leq y^{++} \vee y^{+}$. Consequently,

as required.

$$
\begin{aligned}
x^{\circ 0} \wedge x^{+} & \leq\left(y^{\circ \circ} \vee y^{\circ}\right) \wedge\left(y^{++} \vee y^{+}\right) \\
& =y^{++} \vee\left(y^{\circ 0} \wedge y^{+}\right) \vee y^{\circ} \\
& \leq y^{++} \vee y^{++} \vee y^{\circ} \vee y^{\circ} \text { by (11) } \\
& =y^{++} \vee y^{\circ},
\end{aligned}
$$

Corollary 2.9. Equational bases for $\mathbf{S I}_{2}, \mathbf{S I}_{4}, \mathbf{S I}_{6}, \mathbf{S I}_{7}$ are $(2),(3,5),(\alpha, 4,15)$, $\left(\alpha, 4^{\star}, 15\right)$ respectively.

It is possible to find two-relation equational bases for other subvarieties in this lattice. The following are two examples.

COROLLARY 2.10. An equational basis for $\mathbf{S I}_{3} \vee \mathbf{S I}_{8} \vee \mathbf{S I}_{10}$ is $(\alpha, 7)$. 
Proof. Observe that (7) gives $x^{\circ \circ} \wedge x^{\circ} \leq x \vee y \vee y^{\circ}$ and hence $x^{\circ \circ} \wedge x^{\circ} \leq x^{++} \vee y^{++} \vee$ $y^{\circ}$ from which (12) follows. It also gives $x^{\circ} \wedge x^{\circ} \leq x^{++} \vee y^{\circ \circ} \vee y^{\circ}$ from which (9') follows on applying ${ }^{\circ}$ and interchanging $x$ and $y$. It suffices, therefore, to show that $\left(9^{\prime}\right)$ and (12) together imply (7). For this purpose, note that on writing $x^{++}$for $x$ in $\left(9^{\prime}\right)$, then applying + , then interchanging $x$ and $y$, we obtain

$$
x^{++} \vee y^{++} \vee y^{+} \geq x^{\infty} \wedge x^{\circ} .
$$

Using this and (12), we then have

$$
\begin{aligned}
x^{++} \vee y^{++} \vee y^{\circ} & =x^{++} \vee y^{++} \vee y^{\circ} \vee\left(x^{\circ \circ} \wedge x^{\circ} \wedge y^{+}\right) \\
& =\left(x^{\circ \circ} \vee y^{++} \vee y^{\circ}\right) \wedge\left(x^{++} \vee x^{\circ} \vee y^{++} \vee y^{\circ}\right) \wedge\left(x^{++} \vee y^{++} \vee y^{+}\right) \\
& \geq x^{\circ \circ} \wedge x^{\circ} \wedge x^{\circ} \wedge x^{\circ} \\
& =x^{\circ \circ} \wedge x^{\circ} .
\end{aligned}
$$

It follows that

$$
x^{\circ} \wedge x^{\circ} \leq\left(x^{++} \vee y^{++} \vee y^{\circ}\right) \wedge x^{\circ} \leq\left(x^{++} \wedge x^{\circ}\right) \vee y^{++} \vee y^{\circ}
$$

and hence that $\left(x^{\circ \circ} \wedge x^{\circ}\right) \vee y \vee y^{\circ}=\left(x^{++} \wedge x^{\circ}\right) \vee y \vee y^{\circ}$ from which (7) follows.

Corollary 2.11. An equational basis for $\mathbf{S I}_{3} \vee \mathbf{S I}_{10}$ is $(\alpha, 5)$.

Proof. In [6] we showed that (6) $\wedge$ (7) is (5).

Of course, equational bases are not unique and it is possible to derive other two-relation bases. By way of example:

Corollary 2.12. An equational basis for $\mathbf{S I}_{3} \vee \mathbf{S I}_{10}$ is $(\alpha, 16)$.

Proof. Observe that if in $(\alpha)$ we write $x \wedge x^{+}$for $x$ and $y \vee y^{\circ}$ for $y$ then we obtain

$$
x \wedge x^{+} \wedge\left(y^{\circ \circ} \vee y^{\circ}\right) \leq\left(x^{++} \wedge x^{+}\right) \vee y \vee y^{\circ} .
$$

Now by (5) and Lemma 2.2 the right-hand side of $\left(^{*}\right)$ reduces to $y \vee y^{\circ}$. Since (5) is self-dual, we have $x \wedge x^{+} \leq y \vee y^{+}$, equivalent to $x \wedge x^{+} \leq y^{\circ} \vee y^{\circ}$, and so the left-hand side of $\left(^{\star}\right)$ reduces to $x \wedge x^{+}$.

3. Locally convex skeletons. We shall say that a double MS-algebra has a locally convex skeleton if every interval sublattice of the form $\left[x^{\circ}, x^{+}\right]$belongs to the skeleton.

TheORem 3.1. Let $\left(L,{ }^{\circ},{ }^{+}\right)$be a double MS-algebra. Then the following statements are equivalent:

$\left(\beta_{0}\right) L$ has a locally convex skeleton;

(B) $(\forall x, y \in L) \quad x^{\circ} \wedge y^{+} \leq x^{++} \vee \cdot y^{\circ}$;

$\left(\beta_{1}\right) L \in \mathbf{S I}_{20}$.

Proof. $\left(\beta_{0}\right) \Leftrightarrow(\beta)$ : Clearly, $z \in\left[y^{\circ}, y^{+}\right]$if and only if $z$ is of the form $\left(x \vee y^{\circ}\right) \wedge y^{+}$, 
and this is in the skeleton if and only if

$$
\left(x^{\infty} \vee y^{\circ}\right) \wedge y^{+}=\left(x^{++} \vee y^{\circ}\right) \wedge y^{+},
$$

which is equivalent to

$$
\left(x^{\infty \circ} \wedge y^{+}\right) \vee y^{\circ}=\left(x^{\circ} \vee y^{\circ}\right) \wedge y^{+} \leq x^{++} \vee y^{\circ},
$$

which is equivalent to $(\beta)$.

$(\beta) \Leftrightarrow\left(\beta_{1}\right)$ : It suffices to check, with reference to Fig. 1 , that $(\beta)$ is satisfied by $S I_{20}$ but is not satisfied by $\mathrm{SI}_{10}$ or $S I_{3}$.

It follows from Theorem 3.1 that the double MS-algebras having locally convex skeletons form a variety, namely $\mathbf{S I}_{20}$.

As before, we can construct its lattice of subvarieties. This has 25 elements and is depicted in Fig. 4.

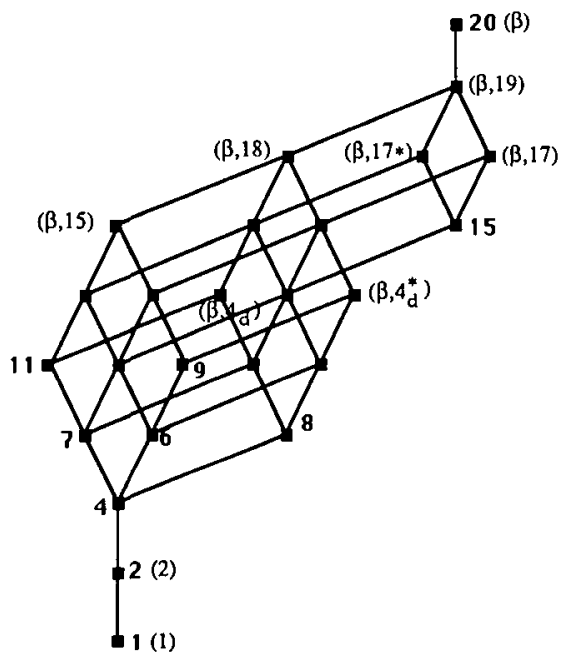

Figure 4.

As for equational bases, we apply the same technique as before, determining first equational bases for the $\wedge$-irreducible elements in the lattice. For this purpose, consider the following relations:

$$
\begin{aligned}
& \text { (1), (2), }\left(4_{d}\right),\left(4_{d}^{\star}\right),(15) \\
& \text { (17) } x \wedge x^{+} \wedge y^{\circ} \leq x^{++} \vee y, \\
& \left(17^{\star}\right) x \wedge y^{\circ \circ} \leq x^{++} \vee y \vee y^{\circ}, \\
& \text { (18) } x^{\circ \circ} \wedge x^{+} \wedge y^{\circ} \leq x^{++} \vee y^{++} \vee y^{\circ}, \\
& \text { (19) } x \wedge x^{+} \wedge y^{\circ 0} \leq x^{++} \vee y \vee y^{\circ}, \\
& (\beta)=(14) x^{\circ} \wedge y^{+} \leq x^{++} \vee y^{\circ} .
\end{aligned}
$$


Under logical implication, these relations give rise to the Hasse diagram of Fig. 5.

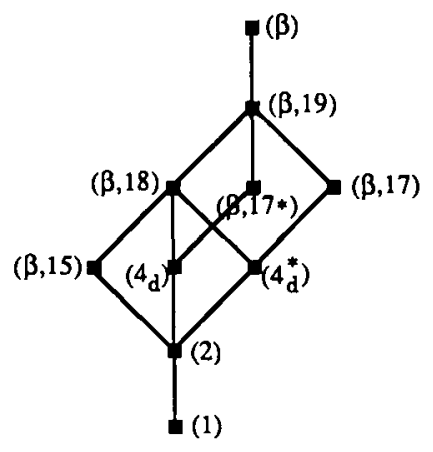

Figure 5.

Obviously, (18) and (19) are self-dual; and in Fig. 5 all of the implications are clear except for $\left(4_{d}\right) \Rightarrow(18),(2) \Rightarrow(\beta)$, and $\left(4_{d}\right) \Rightarrow(\beta)$.

Now if $\left(4_{d}\right)$ holds then $y^{\infty} \leq y^{\circ} \vee y^{\circ}=y \vee y^{\circ}=y^{++} \vee y^{\circ}$ whence (18) follows.

As for $(2) \Rightarrow(\beta)$, it suffices to observe that (2) implies (3) which is equivalent to $x^{\infty}=x^{++}$.

To see that $\left(4_{d}\right) \Rightarrow(\beta)$, recall from [6] that $\left(4_{d}\right)$ is equivalent to

$\left(4_{d}^{\prime \prime}\right) \quad x \vee y \vee y^{\circ}=x^{\infty} \vee y \vee y^{\circ}$.

It follows from these that

$$
x^{\infty} \leq x^{++} \vee y \vee y^{\circ} .
$$

But, as we have seen, $\left(4_{d}\right)$ also gives $y^{\circ} \leq y \vee y^{\circ}$ whence we have $y^{\circ} \geq y^{+} \wedge y^{\infty}$ and consequently

These observations give

$$
y^{\circ}=y^{+} \wedge y^{\infty} \geq y^{+} \wedge y
$$

$$
\begin{aligned}
x^{\infty} \wedge y^{+} & \leq\left(x^{++} \vee y \vee y^{\circ}\right) \wedge y^{+} \\
& =\left(x^{++} \wedge y^{+}\right) \vee\left(y \wedge y^{+}\right) \vee y^{\circ} \\
& \leq x^{++} \vee y^{\circ}
\end{aligned}
$$

which is $(\beta)$.

Using Figs 4 and 5, we can argue as in Theorem 2.5 to obtain the following result.

THEOREM 3.2. In the lattice of subvarieties of double MS-algebras having locally convex skeletons the $\wedge$-irreducible elements have the following equational bases:

$$
\begin{array}{ll}
\mathbf{S I}_{1}:(1), & \mathbf{S I}_{2}:(2), \\
\mathbf{S I}_{8} \vee \mathbf{S I}_{9}:\left(4_{d}^{\star}\right), & \mathbf{S I}_{8} \vee \mathbf{S I}_{11}:\left(4_{d}\right), \\
\mathbf{S I}_{9} \vee \mathbf{S I}_{11}:(\beta, 15), & \mathbf{S I}_{9} \vee \mathbf{S I}_{15}:(\beta, 17), \\
\mathbf{S I}_{11} \vee \mathbf{S I}_{15}:\left(\beta, 17^{\star}\right), & \mathbf{S I}_{8} \vee \mathbf{S I}_{9} \vee \mathbf{S I}_{11}:(\beta, 18), \\
\mathbf{S I}_{9} \vee \mathbf{S I}_{11} \vee \mathbf{S I}_{15}:(\beta, 19), & \mathbf{S I}_{20}:(\beta) .
\end{array}
$$


COROLlaRY 3.3. Equational bases for $\mathbf{S I}_{11}, \mathbf{S I}_{9}$ are $\left(4_{d}, 15\right)$, $\left(4_{d}^{\star}, 15\right)$ respectively.

4. Kleene skeletons. We shall now turn our attention to the subvariety $\mathbf{S I}_{18} \vee \mathbf{S I}_{19}$. We begin by identifying this as the subvariety consisting of those MS-algebras whose skeleton is a Kleene algebra.

THEOREM 4.1. On a double MS-algebra $\left(L,{ }^{\circ},+\right)$ the following conditions are equivalent:

$\left(\gamma_{0}\right)$ L has a Kleene skeleton;

$(\gamma)(\forall x, y \in L) \quad x \wedge x^{\circ} \leq y \vee y^{+}$

$\left(\gamma_{1}\right) L \in \mathbf{S I}_{18} \vee \mathbf{S I}_{19}$.

Proof. $\left(\gamma_{0}\right)$ holds if and only if $x^{\infty} \wedge x^{\circ} \leq y^{\infty} \vee y^{\circ}$ and by Lemma 2.2 this is equivalent to $(\gamma)$. The equivalence of $(\gamma)$ and $\left(\gamma_{1}\right)$ results from the fact that only the subdirectly irreducibles contained in $S I_{18}$ or $S I_{19}$ satisfy $(\gamma)$.

The lattice of subvarieties of double MS-algebras with a Kleene skeleton can be constructed using the same technique as before. The lattice in question has 99 elements and can be visualised using Figs. $6 \mathrm{a}, 6 \mathrm{~b}, 6 \mathrm{c}$. Add Fig. $6 \mathrm{~b}$ as a second layer on top of Fig. 6a projecting down onto it, with $\mathbf{S I}_{3}$ directly above $\mathbf{S I}_{2}$, then Fig. $6 \mathrm{c}$ as a third layer projecting down onto Fig. $6 \mathrm{~b}$ with $\mathbf{S I}_{5}$ directly above $\mathbf{S I}_{3}$.

To determine equational bases in this case, we consider the following relations:

$$
\begin{aligned}
& \text { (1), (2 } \left.d_{d}\right),(4),\left(4^{\star}\right),(10),(11),(12),(19) \text {, } \\
& \text { (20) } x \wedge y^{\circ 0} \leq x^{++} \vee y \vee y^{+} \text {, } \\
& \text { (20") } x \wedge x^{\circ} \wedge y^{\circ 0} \leq x^{++} \vee y \text {, } \\
& \text { (21) } x \wedge y^{\circ} \wedge z^{+} \leq x^{++} \vee y \vee y^{+} \vee z^{\circ} \text {, } \\
& \text { (21 }) x \wedge x^{\circ} \wedge y^{\infty} \wedge z^{+} \leq x^{++} \vee y \vee z^{\circ} \text {, } \\
& \text { (22) } x \wedge y^{\infty} \wedge z^{\infty} \wedge z^{+} \leq x^{++} \vee y \vee y^{+} \vee z^{\circ} \text {, } \\
& \text { (22*) } x \wedge x^{\circ} \wedge y^{\infty} \wedge z^{+} \leq x^{++} \vee y \vee z^{++} \vee z^{\circ} \text {, } \\
& \text { (23) } x \wedge y^{\circ 0} \wedge z \wedge z^{+} \leq x^{++} \vee y \vee y^{+} \vee z^{\circ} \text {, } \\
& \left(23^{\star}\right) x \wedge x^{\circ} \wedge y^{\circ} \wedge z^{+} \leq x^{++} \vee y \vee z \vee z^{\circ} \text {, } \\
& (\gamma)=(6) \cdot x \wedge x^{\circ} \leq y \vee y^{+} \text {. }
\end{aligned}
$$

Ordered by implication, these relations give rise to the Hasse diagram of Fig. 7 .

Of the implications in Fig. 7, the following are non-trivial:

$(10) \Rightarrow\left(21^{\star}\right)$ : By (10), $x^{\circ} \wedge x^{\circ} \wedge z^{+} \leq x^{++} \vee z^{\circ}$ whence $x \wedge x^{\circ} \wedge z^{+} \leq x^{++} \vee z^{\circ}$ and $\left(21^{\star}\right)$ follows.

$(12) \Rightarrow\left(22^{\star}\right)$ : By (12), $x^{\circ} \wedge x^{\circ} \wedge z^{+} \leq x^{++} \vee z^{++} \vee z^{\circ}$ whence $x \wedge x^{\circ} \wedge z^{+} \leq x^{++} \vee$ $z^{++} \vee z^{\circ}$ and $\left(22^{\star}\right)$ follows.

$(12, \gamma) \Rightarrow(22)$ : By Corollary $2.11,(12, \gamma)=(12,6)$ is equivalent to $x \wedge x^{\circ} \leq y \vee y^{\circ}$. Applying ${ }^{+}$to this, we obtain $y^{+} \wedge y^{\infty} \leq x^{+} \vee x^{\infty}$. Writing $x^{++}$for $x$, we obtain

$$
y^{+} \wedge y^{\infty} \leq x^{+} \vee x^{++} \leq x^{+} \vee x .
$$


EQUATIONAL BASES FOR SUBVARIETIES
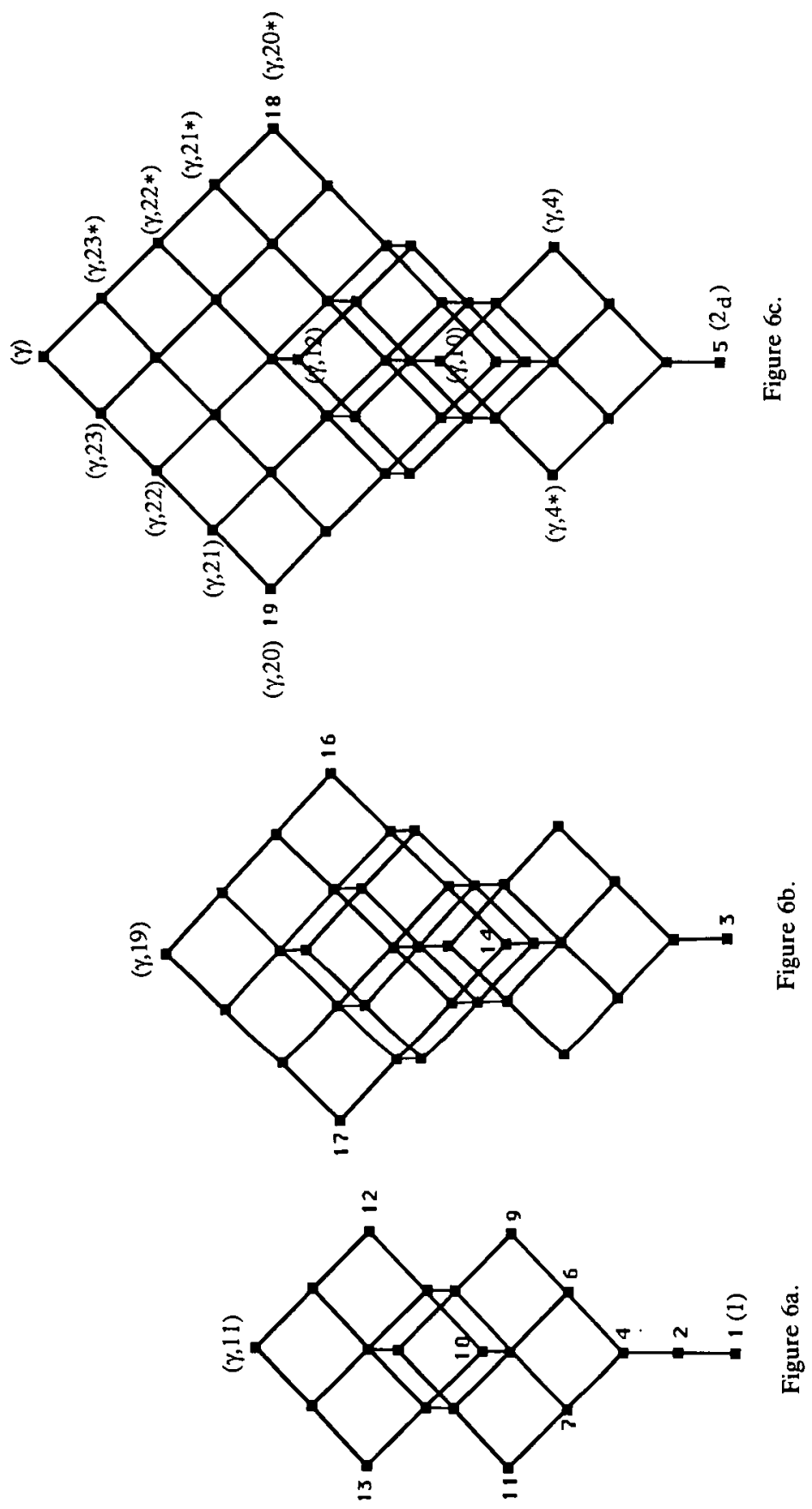


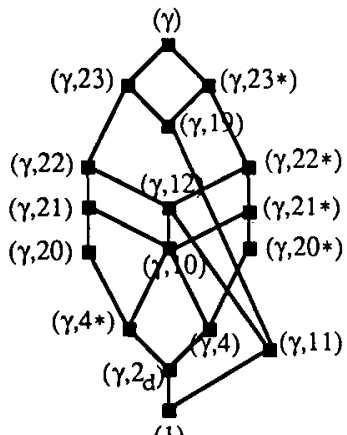

(1)

Figure 7.

Now write $z$ for $y$ and $y$ for $x$ to obtain $z^{+} \wedge z^{\infty} \leq y^{+} \vee y$ from which (22) follows.

$(11, \gamma) \Rightarrow(19)$ : By Corollary $2.8,(11, \gamma)$ is equivalent to $x^{\infty} \wedge x^{+} \leq y^{++} \vee y^{\circ}$. This gives $x \wedge x^{+} \leq y \vee y^{\circ}$ from which (19) follows.

(19) $\Rightarrow$ (23): Writing $x \wedge z$ for $x$ in (19), we obtain

$$
x \wedge z \wedge\left(x^{+} \vee z^{+}\right) \wedge y^{\infty} \leq\left(x^{++} \wedge z^{++}\right) \vee y \vee y^{\circ} .
$$

Consequently, $x \wedge y^{\infty} \wedge z \wedge z^{+} \leq x^{++} \vee y \vee y^{\circ}$ which implies (23).

THEOREM 4.2. In the lattice of subvarieties of double MS-algebras with a Kleene skeleton the $\wedge$-irreducible elements have the following equational bases:

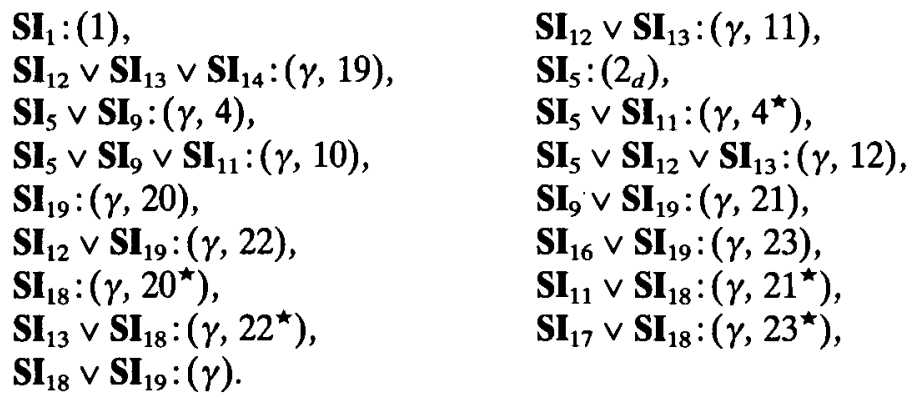

CoRollaRY 4.3. Equational bases for $\mathbf{S I}_{17}$ and $\mathbf{S I}_{16}$ are $\left(\gamma, 17^{\star}\right)$ and $(\gamma, 17)$ respectively.

Proof. It suffices to prove that (19) and (20) together imply $\left(17^{*}\right)$. Now, writing $y \vee x^{+}$for $y$ in (20) we have $x \wedge y^{\circ} \leq x^{++} \vee x^{+} \vee y$. Using this and (19), we then have

$$
\begin{aligned}
x^{++} \vee y \vee y^{\circ} & =x^{++} \vee y \vee y^{\circ} \vee\left(x \wedge x^{+} \wedge y^{\infty}\right) \\
& =\left(x \vee y \vee y^{\circ}\right) \wedge\left(x^{++} \vee x^{+} \vee y \vee y^{\circ}\right) \wedge\left(x^{++} \vee y^{\infty} \vee y^{\circ}\right) \\
& \geq x \wedge\left(x \wedge y^{\infty}\right) \wedge y^{\infty} \\
& =x \wedge y^{\infty},
\end{aligned}
$$

as required. 
Corollary 4.4. Equational bases for $\mathbf{S I}_{13}$ and $\mathbf{S I}_{12}$ are $\left(15,17^{\star}\right)$ and $(15,17)$ respectively.

Proof. For example, $\mathbf{S I}_{13}=\left(\mathbf{S I}_{12} \vee \mathbf{S I}_{13}\right) \wedge \mathbf{S I}_{17}$ and by Theorem 4.2 an equational basis for $\mathbf{S I}_{12} \vee \mathbf{S I}_{13}$ is $(\gamma, 11)$ which, as shown in the proof of Corollary 2.8 , is equivalent to (15). The result now follows by Corollary 4.3 .

Further subvarieties that have interesting equational bases are the following, the first two of which have been noted above.

Corollary 4.5. An equational basis for $\mathbf{S I}_{12} \vee \mathbf{S I}_{13}$ is (15).

Corollary 4.6. An equational basis for $\mathbf{S I}_{5} \vee \mathbf{S I}_{12} \vee \mathbf{S I}_{13}$ is (5).

COROLlaRy 4.7. An equational basis for $\mathbf{S I}_{3} \vee \mathbf{S I}_{12} \vee \mathbf{S I}_{13}$ is (16).

Proof. Observe that since

$$
\mathbf{S I}_{3} \vee \mathbf{S I}_{12} \vee \mathbf{S I}_{13}=\left(\mathbf{S I}_{5} \vee \mathbf{S I}_{12} \vee \mathbf{S I}_{13}\right) \wedge\left(\mathbf{S I}_{12} \vee \mathbf{S I}_{13} \vee \mathbf{S I}_{14}\right)
$$

it suffices to show that $(\gamma),(12),(19)$ together imply (16). Now we have observed above that $(\gamma, 12)$ is equivalent to $x \wedge x^{\circ} \leq y \vee y^{\circ}$. It follows from this that $x^{\circ} \wedge x^{\circ} \wedge y^{+} \leq y^{++} \vee$ $y^{\circ}$. Applying ${ }^{\circ}$ to this, then interchanging $x$ and $y$, we obtain $x^{\infty} \wedge x^{+} \leq x^{++} \vee y^{\infty} \vee y^{\circ}$. This, together with (19), gives

$$
\begin{aligned}
x^{++} \vee y \vee y^{\circ} & =x^{++} \vee y \vee y^{\circ} \vee\left(x \wedge x^{+} \wedge y^{\infty}\right) \\
& =\left(x \vee y \vee y^{\circ}\right) \wedge\left(x^{++} \vee x^{+} \vee y \vee y^{\circ}\right) \wedge\left(x^{++} \vee y^{\infty} \vee y^{\circ}\right) \\
& \geq x \wedge x^{+} \wedge x^{\infty} \wedge x^{+} \\
& =x \wedge x^{+} .
\end{aligned}
$$

Consequently, $\left(x \wedge x^{+}\right) \vee y \vee y^{\circ}=\left(x^{++} \wedge x^{+}\right) \vee y \vee y^{\circ}=y \vee y^{\circ}$, by Theorem 2.2, and hence $x \wedge x^{+} \leq y \vee y^{\circ}$.

5. Concluding remarks. From the above results we can compile the following list of equational bases for the subvarieties generated by the non-trivial subdirectly irreducible double MS-algebras.

$$
\begin{array}{llll}
\mathbf{S I}_{2}:(2), & \mathbf{S I}_{3}:\left(\alpha, 2_{d}\right), & \mathbf{S I}_{4}:(3,5), & \mathbf{S I}_{5}:\left(2_{d}\right), \\
\mathbf{S I}_{6}:(\alpha, 4,15), & \mathbf{S I}_{7}:\left(\alpha, 4^{\star}, 15\right), & \mathbf{S I}_{8}:(3), & \mathbf{S I}_{9}:\left(4_{d}^{\star}, 5\right), \\
\mathbf{S I}_{10}:(\alpha, 15), & \mathbf{S I}_{11}:(4 d, 5), & \mathbf{S I}_{12}:(15,17), & \mathbf{S I}_{13}:\left(15,17^{\star}\right), \\
\mathbf{S I}_{14}:(\alpha, \gamma), & \mathbf{S I}_{15}:(\alpha, \beta), & \mathbf{S I}_{16}:(\gamma, 17), & \mathbf{S I}_{17}:\left(\gamma, 17^{\star}\right), \\
\mathbf{S I}_{18}:\left(\gamma, 20^{\star}\right), & \mathbf{S I}_{19}:(\gamma, 20), & \mathbf{S I}_{20}:(\beta), & \mathbf{S I}_{21}:(\alpha) .
\end{array}
$$

Of the above classes, five are worthy of especial mention, namely $\mathbf{S I}_{2}, \mathbf{S I}_{\mathbf{4}}, \mathbf{S I}_{\mathbf{8}}, \mathbf{S I}_{5}, \mathbf{S I}_{3}$. These are respectively the classes of boolean, Kleene, de Morgan, double Stone, and three-valued Lukasiewicz algebras. The first four assertions are clear since the axioms (2), $\left(2_{d}\right),(3),(\gamma)$ are self-dual. As for the final assertion:

THEOREM 5.1. $\mathrm{SI}_{3}$ is the class of three-valued Lukasiewicz algebras. 
Proof. A three-valued Lukasiewicz algebra is a double Stone algebra $\left(L,^{\star},{ }^{+}\right)$which satisfies the Moisil determination principle $\Phi_{+}^{\star}=\omega$; i.e. an algebra in $\mathbf{S I}_{5}$ which is semisimple, i,e. an algebra in $\mathbf{S I}_{\mathbf{3}}$.

Corollary 5.2. An equational basis for $\mathrm{SI}_{3}$ is $\left(2_{d}, 16\right)$.

Proof. In the lattice of subvarieties we have

$$
\mathbf{S I}_{3}=\left(\mathbf{S I}_{3} \vee \mathbf{S I}_{12} \vee \mathbf{S I}_{13}\right) \wedge \mathbf{S I}_{5},
$$

so the result follows by Corollary 4.7.

\section{REFERENCES}

1. R. Balbes and P. Dwinger, Distributive lattices, (University of Missouri Press, 1974).

2. R. Beazer, On some small varieties of distributive Ockham algebras, Glasgow Math. Journal, 25 (1984), 175-181.

3. J. Berman, Distributive lattices with an additional unary operation, Aequationes Math., 16 (1977), 165-171.

4. Joel Berman and Peter Köhler, Finite distributive lattices and finite partially ordered sets, Mitt. Math. Sem. Giessen, 121 (1976), 103-124.

5. T. S. Blyth and J. C. Varlet, On a common abstraction of de Morgan algebras and Stone algebras, Proc. Roy. Soc. Edinburgh, 94A (1983), 301-308.

6. T. S. Blyth and J. C. Varlet, Subvarieties of the class of MS-algebras, Proc. Roy. Soc. Edinburgh, 95A (1983), 157-169. 37-47.

7. T. S. Blyth and J. C. Varlet, Double MS-algebras, Proc. Roy. Soc. Edinburgh, 98A (1984),

8. T. S. Blyth and J. C. Varlet, Subdirectly irreducible double MS-algebras, Proc. Roy. Soc. Edinburgh, 98A (1984), 241-247.

9. S. Burris and H. P. Sankappanavar, A course in universal algebra, Graduate Texts in Mathematics 78 (Springer Verlag Berlin, 1981).

10. W. H. Cornish, Antimorphic Action, Research and Exposition in Mathematics, 12 (Heldermann Verlag Berlin, 1986).

11. B. A. Davey, On the lattice of subvarieties, Houston J. Math., 5 (1979), 183-192.

12. G. Grätzer, General Lattice Theory, (Birkhäuser Verlag Basel, 1978).

13. B. Jónsson, Algebras whose congruence lattices are distributive, Math. Scand., 21 (1967), $110-121$.

14. A. S. A. Noor, Bistable subvarieties of MS-algebras, Proc. Roy. Soc. Edinburgh, 105A (1987), 127-128.

15. H. P. Sankappanavar, Distributive lattices with a dual endomorphism, Zeitschr. f. math. Logik und Grundlagen d. Math., 31 (1985), 385-392.

Mathematical Institute,

UNIVERSITY OF ST ANDREWS, ST ANDREWS KY16 9SS,

FIFE, SCOTLAND.

Department of Mathematics,

RAJSHAHI UNIVERSITY,

RAJSHAHI,

BANGLADESH.
INSTITUT DE Mathematique, UNIVERSITE DE LIEGE, AVenue des Tilleuls, 15 B-400 Liege, Belgique. 